\title{
Pourquoi les logisticiens doivent penser " traçabilité totale " des Supply Chains
}

\author{
La traçabilité des produits dans les chaînes logistiques est indispensable pour garantir \\ l'origine des produits, organiser le rappel de produits défectueux ou attester de la qualité \\ du processus global de leur production-distribution jusqu'au consommateur final \\ (et plus globalement de tout leur cycle de vie).A l'heure où l'électronique s'embarque \\ dans tous les matériels, où les objets connectés se multiplient, où les individus \\ (salariés, consommateurs, citoyens) sont « en ligne » en permanence via leur ordinateur, \\ leur tablette ou leur mobile, le champ de la traçabilité s'étend et ouvre de nouvelles \\ perspectives pour passer d'une traçabilité des produits dans les Supply Chains à \\ une traçabilité totale des Supply Chains.
}

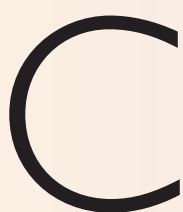

ette traçabilité « totale " présente un intérêt majeur pour la logistique, à condition que les logisticiens, souvent mis à contribution pour développer et mettre en œuvre les systèmes de traçabilité, les intègrent pleinement dans leurs systèmes de pilotage, et pensent en termes de traçabilité " totale " pour la logistique. Une traçabilité totale des produits, processus et activités, mais aussi des ressources disponibles et mobilisables ainsi que du contexte de déploiement des chaînes, permet en effet de s'assurer que tout se passe comme prévu et crée les " valeurs " attendues. Elle permet aussi de réagir en cas d'aléa et de capitaliser les expériences pour mieux prévoir et planifier les futures activités. Elle apparaît donc indispensable au pilotage des Supply Chains. Cette traçabilité totale, qui représente un « idéal " pour les logisticiens - qui rêvent d'avoir de la visibilité sur leur Supply Chain -, n'est cependant pas si simple à concevoir et mettre en œuvre.

\section{La traçabilité totale des Supply Chains :} des défis techniques et organisationnels La plupart des travaux sur la traçabilité renvoient en effet à une approche largement intra-organisationnelle

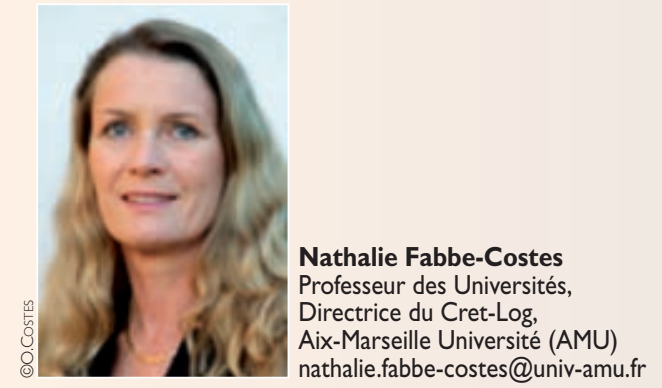

de la traçabilité (tracer les flux dans l'entreprise), et ne considèrent que les interfaces amont et aval avec les partenaires directs de l'entreprise (l'accrochage de proche en proche des systèmes de traçabilité internes des entreprises). La traçabilité totale dépasse cette vision et envisage la traçabilité sur l'ensemble de la Supply Chain (voir Figure 1). Le caractère inter-organisationnel de cette vision de la traçabilité (voir tableau 1)

Figure I

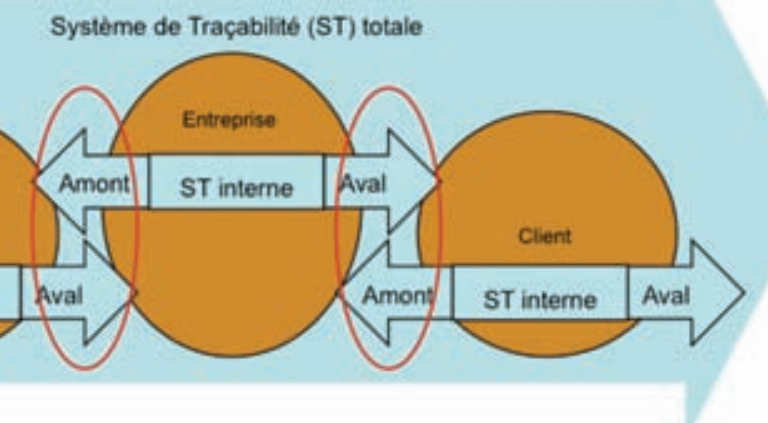

demande une certaine harmonisation, du moins une compatibilité, tant pour la codification de tous les éléments tracés, que pour leur marquage, le captage des données de traçabilité, leur mémorisation et mutualisation. Il soulève au plan technique plusieurs défis en lien avec l'existence de normes ou de standards ${ }^{1}$, la capacité d'interopérabilité et d'inter-connectivité des systèmes, voire le développement de processus et systèmes communs ou partagés. Il soulève aussi au plan organisationnel des défis importants liés à la gestion des données de traçabilité : qui est responsable de la production de quelles données? Qui en garantit la qualité ? Comment s'organise la mise à disposition, le partage, l'accès à ces données ?

La traçabilité totale, un enjeu stratégique dans les Supply Chains

A un niveau plus stratégique, se pose par ailleurs la question de la valeur des informations ainsi partagées,

I. Voir Billet CRET-LOG SCMag n67, septembre 2012 
de l'impact potentiel de la visibilité donnée sur la chaîne logistique, et de l'usage qui sera fait de ces données de traçabilité. Est-il bien raisonnable de travailler en toute "transparence " ? Jusqu'où donner à voir ses flux, processus, ressources ? Mettre en œuvre la traçabilité totale suppose ainsi une réflexion de fond qui ne s'inscrive pas dans une vision collaborative parfois un peu naïve du Supply Chain Management, et ne se focalise pas seulement sur les questions techniques mises en avant par les sociétés proposant des " solutions " de traçabilité (qu'il ne faut certes pas négliger). La traçabilité totale soulève donc des enjeux de pilotage des chaînes logistiques, en lien avec la gouvernance des Supply Chains, ce qui renvoie à une lecture des relations inter-organisationnelles en termes de pouvoir, d'opportunisme ${ }^{2}$ mais aussi de coopétition. Dès lors que le pilotage des chaînes logistiques (désormais mondiales et multi-acteurs) s'appuie sur les données de traçabilité totale, une approche en termes de risk management est par ailleurs indispensable. Quelle confiance peut-on avoir dans ces données ? Comment ne pas se faire voler des données considérées comme stratégiques? Quel est le risque de travailler sur des données volontairement faussées ?

\section{L'évolution permanente du système de traçabilité totale}

Les recherches du Cret-Log sur la traçabilité et la mise en œuvre des systèmes de traçabilité totale depuis le début des années 1990 (à une époque où ce n'était pas à la mode), nous ont aussi permis de mieux comprendre l'évolution des systèmes de traçabilité totale. La concrétisation puis l'évolution de ces systèmes se produisent dans un champ de forces multiples. La diffusion de nouvelles technologies, l'émergence de nouvelles exigences tant règlementaires (ex : en lien avec la logistique durable) que du fait de certains acteurs (internes ou externes aux Supply Chains), expliquent notamment l'évolution des systèmes de traçabilité. A l'inverse, la forte intégration des chaînes logistiques, leur caractère routinier, la focalisation sur les exigences de maîtrise, voire de réduction, des coûts logistiques, ou l'absence de collaboration entre les acteurs, se traduisent par des phénomènes de lock-in et freinent leur évolution. A l'heure où se développe une hyper-traçabilité qui produit ce qui est actuellement appelé le Big Data, il nous semble important que les logisticiens pensent proactivement les systèmes de traçabilité totale et l'usage qu'ils pourront faire des données qu'ils produisent. De nouvelles compétences vont se révéler nécessaires en matière d'analyse de données pour l'aide à la décision logistique... compétences à développer d'urgence !

2. Voir Billet CRET-LOG SCMag n69, novembre 2012

\section{Tableau}

Dimensions de la Composantes du système de traçabilité totale traçabilité totale

I. Identification Codification des entités, activités, acteurs identifiés $\begin{array}{lll}\text { des éléments } & \text { Principe(s) d'identification(s) retenu(s) }\end{array}$

tracés $\quad$ Pertinence de l'identification par rapport aux objectifs de la traçabilité Maille de traçabilité

\begin{tabular}{|c|c|}
\hline \multirow{3}{*}{$\begin{array}{l}\text { 2. Capture } \\
\text { des données } \\
\text { de traçabilité }\end{array}$} & $\begin{array}{l}\text { Supports des codes et modes de lecture } \\
\text { Lecture continue/discontinue }\end{array}$ \\
\hline & $\begin{array}{l}\text { Enregistrement des données de traçabilité } \\
\text { Couplage entre flux, activités, acteurs }\end{array}$ \\
\hline & $\begin{array}{l}\text { Exactitude et fiabilité des données captées } \\
\text { Niveau d'exhaustivité }\end{array}$ \\
\hline \multirow{3}{*}{$\begin{array}{l}\text { 3. Mémorisation } \\
\text { des données } \\
\text { de traçabilité }\end{array}$} & $\begin{array}{l}\text { Stockage (type et capacité) } \\
\text { Structuration du stockage des données de traçabilité }\end{array}$ \\
\hline & $\begin{array}{l}\text { Type d'accès aux données de traçabilité } \\
\text { Disponibilité en temps réel }(\mathrm{O} / \mathrm{N})\end{array}$ \\
\hline & $\begin{array}{l}\text { Durée de stockage des données de traçabilité } \\
\text { Nature des requêtes pour obtenir une information } \\
\text { de traçabilité }\end{array}$ \\
\hline \multirow{2}{*}{$\begin{array}{l}\text { 4. Traitement } \\
\text { et transmission } \\
\text { des données } \\
\text { de traçabilité }\end{array}$} & Traitement des informations de traçabilité \\
\hline & $\begin{array}{l}\text { Pilotage de la transmission } \\
\text { (diffusion automatique ou sur demande) } \\
\text { Périmètre des informations partagées } \\
\text { (en interne et avec les partenaires amont/aval) } \\
\text { Contrôle des accès aux données de traçabilité }\end{array}$ \\
\hline \multirow{3}{*}{$\begin{array}{l}\text { 5. Coordination } \\
\text { intra et inter- } \\
\text { organisationnelle } \\
\text { de la traçabilité }\end{array}$} & $\begin{array}{l}\text { Degré de standardisation/normalisation } \\
\text { Choix et portée des standards/normes } \\
\text { inter-organisationnels retenus }\end{array}$ \\
\hline & $\begin{array}{l}\text { Procédures communes en lien avec la traçabilité } \\
\text { Niveau de formalisation des relations, } \\
\text { types de partenariats } \\
\text { Développement d'actions collectives } \\
\text { en lien avec la traçabilité }\end{array}$ \\
\hline & $\begin{array}{l}\text { Degré de compatibilité des systèmes de traçabilité } \\
\text { Interopérabilité et interconnectivité des systèmes } \\
\text { de traçabilité } \\
\text { Nature et niveau d'intégration des organisations } \\
\text { et des systèmes d'information }\end{array}$ \\
\hline
\end{tabular}

\section{Pour en savoir plus}

Trois thèses ont été soutenues sur ce sujet, une thèse en cours sera soutenue fin 2014 .

Plusieurs publications du Cret-Log sur la traçabilité peuvent être consultées.

Une synthèse récente vient d'être publiée aux Techniques de l'Ingénieur :Traçabilité et logistique : les interactions, N. Fabbe-Costes, Encyclopédie « Techniques de l'Ingénieur », traité « Traçabilité », Ref. Doc. TR 300v2, Ed. Techniques de L'Ingénieur, Paris, Ed. I I-20I3.

Un chapitre d'ouvrage sur l'hyper-traçabilité est à paraître.

Le Cret-Log est un centre de recherche en sciences de gestion d'Aix Marseille Université spécialisé en logistique et Supply Chain Management. www.cret-log.com 